\title{
Liraglutide is a Perioperative Therapeutic Option for Patients with Type 2 Diabetes that Undergo Elective Surgery
}

Naoko Katagiri ${ }^{1}$, Ikue Kigawa ${ }^{2}$, Yumiko Yahara ${ }^{1}$, Yohei Ueda ${ }^{1}$, Kumiko Hamano ${ }^{2}$ and Shizuka Kaneko ${ }^{{ }^{*}}$

${ }^{1}$ Division of Diabetes/Endocrinology/Lifestyle-Related Disease, Takatsuki Red Cross Hospital, Japan

${ }^{2}$ Kanto Rosai Hospital, Japan

\begin{abstract}
Diabetic patients that perform limited amounts of exercise during perioperative periods tend to gain weight, and their glycemic control frequently deteriorates. However, temporary insulin therapy is associated with a risk of hypoglycemia. To investigate the efficacy and safety of preoperative liraglutide therapy, we performed a retrospective analysis of the cases of Japanese type 2 diabetes mellitus (T2DM) patients that underwent elective surgery.

Liraglutide therapy was initiated in patients with T2DM prior to elective surgery. Regular insulin was also administered in cases in which hyperglycemia (plasma glucose $>200 \mathrm{mg} / \mathrm{dl}$ ) was detected during operations. The glycemic level, the insulin dose during the perioperative period, body mass index (BMI), and perioperative complications were analyzed.

Seventy-three patients with T2DM (male/female: 40/33, age: $69.5 \pm 11.0$, glycated hemoglobin (HbA1c): $7.8 \pm 1.3$, BMI: $26.6 \pm 4.1$ ) were administered liraglutide therapy before undergoing elective surgery; i.e., orthopedic operations (40 cases); cardiac catheterization for ischemic heart disease(12 cases); ophthalmological operations, including procedures for cataracts (13 cases); prostatectomy (2 cases); mastectomy (1 case); unilateral nephrectomy (1 case); total hysterectomy (1 case); total bladder extirpation (1 case); plastic surgery (1 case); and angioplasty for a hemodialysis shunt (1 case).

The preoperative administration of liraglutide therapy resulted in reductions in body weight (mean: -3.1 $\pm 2.0 \mathrm{~kg}$ ) and good glycemic control throughout the perioperative period. Additional regular insulin was only required in 11 patients, who were prescribed $\leq 10$ units each. No hypoglycemic episodes, delayed wound healing, or other complications were observed. The patients' HbAlc levels remained within the normal range (mean: $6.8 \pm 0.8 \%$ ) at 6 postoperative months. Liraglutide represents an effective therapeutic option for safely achieving good glycemic control in perioperative patients with T2DM, especially those who have a limited exercise ability or are at risk of hypoglycemia.
\end{abstract}

\section{Introduction}

Diabetic patients that remain in a sedentary state during the perioperative period tend to gain weight, and their glycemic control frequently deteriorates. There is substantial evidence linking hyperglycemia in surgical patients with unfavorable outcomes. On the other hand, temporary insulin therapy requires frequent monitoring of blood glucose levels and carries a risk of hypoglycemia. A recent large study into this topic (the NICE-SUGAR study) [1] suggested that intensive insulin therapy significantly increases the risk of hypoglycemia and does not have any beneficial effects on the overall mortality rate among critically ill patients.

Analogues of incretins, which are glucagon-like peptide-1 (GLP-1) receptor agonists, are widely used to treat type 2 diabetes (T2DM) since they do not tend to induce weight gain and are associated with a low risk of hypoglycemia [2]. But data about the therapeutic application of these drugs instead of insulin for perioperative glycemic control are scarce [3-5]. To investigate the efficacy and safety of the perioperative use of liraglutide therapy, a GLP-1 receptor agonist, as a non-insulin agent for achieving perioperative glycemic control, we performed a retrospective analysis of the cases of patients with T2DM who underwent elective surgery at two separate hospitals.

\section{Methods}

A retrospective analysis of a total of 86 elective surgical procedures performed on 73 patients with T2DM at two separate hospitals between October 2010 and October 2013 was carried out.

As liraglutide decreases gastrointestinal motility, cases involving gastrointestinal surgery were not included.

\section{Publication History:}

Received: March 04, 2015

Accepted: June 13, 2016

Published: June 15, 2016

\section{Keywords:}

GLP-1 receptor agonist, Liraglutide, Elective surgery, Periopetative period, Glycemic control

\section{Treatment protocol}

Informed consent was obtained from all patients, and the liraglutide therapy was initiated after the cessation of oral antidiabetic drugs.

In cases in which hyperglycemia occurred during the perioperative period, regular insulin was added to the therapy if necessary.

The preoperative target blood glucose level ranged from 140 to $160 \mathrm{mg} / \mathrm{dl}$ (the 2-hour post-prandial blood glucose level), and during operations it ranged from 150 to $250 \mathrm{mg} / \mathrm{dl}$. In the cases involving hyperglycemia, regular insulin was added at a dose that was appropriate to the patient's blood glucose level before each meal (Figure 1).

\section{Postoperative period}

Liraglutide was re-administered, beginning at a dose of $0.6 \mathrm{mg}$ and increasing to the maximum dose, when meals were restarted.

"Corresponding Author: Dr. Shizuka Kaneko, Department of Diabetes/ Endocrinology/Life-related disease, Takatsuki Red Cross Hospital, Japan, E-mail: skayamoe@takatsuki.jrc.or.jp

Citation: Katagiri N, Kigawa I, Yahara Y, Ueda Y, Kaneko S, et al. (2016) Liraglutide is a Perioperative Therapeutic Option for Patients with Type 2 Diabetes that Undergo Elective Surgery. Int J Diabetes Clin Diagn 3: 117. doi: http://dx.doi.org/10.15344/2394-1499/2016/117

Copyright: (c) 2016 Katagiri et al. This is an open-access article distributed under the terms of the Creative Commons Attribution License, which permits unrestricted use, distribution, and reproduction in any medium, provided the original author and source are credited. 


\section{A) Admission Operation (Day 0) Day 3}

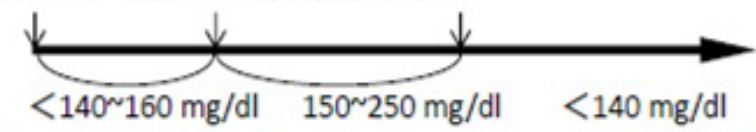

Target blood glucose level (2-hour post-prandial blood glucose level)

B) In cases of hyperglycemia:

\begin{tabular}{cc} 
Glucose (mg/dl) & Additional regular insulin(units) \\
\hline $201 \sim 250$ & 4 \\
$251 \sim 300$ & 6 \\
$301 \sim 350$ & 8 \\
$351 \sim$ & 10
\end{tabular}

\section{C) After operation:}

Lira was re-administered, beginning at a dose of $0.6 \mathrm{mg}$, when meals were started.

Figure 1 Treatment protocol

The target blood glucose level (2-hour post-prandial blood glucose level) ranged from 140 to $160 \mathrm{mg} / \mathrm{dl}$ before the operation and from 150 to $250 \mathrm{mg} / \mathrm{dl}$ after the operation (A). In cases involving hyperglycemia, regular insulin was administered at a dose that was appropriate to the patient's blood glucose level before each meal (B).

Body mass index (BMI), blood glucose levels, hypoglycemia, the insulin dose (if required), the medication administered before the liraglutide therapy, and perioperative complications were analyzed.

In the patients who underwent ophthalmological operations, no glycemic measurements were obtained on the day on which the liraglutide therapy was initiated, the day before the operation, or the day after the operation because these patients were admitted on the day of the operation and discharged the next morning. In addition, the glycemic level was not measured on the day of the operation in the patients that underwent cardiac catheterization.

Data are presented as mean \pm SD. The Student's t-test was employed for statistical analyses.

\section{Results}

\section{Patients}

Seventy-three patients (males: 40) with T2DM underwent liraglutide therapy between October 2010 and October 2013 at two clinical institutions (Table 1).

The patients' baseline characteristics were as follows: male/female, 40/33; mean age, $69.5 \pm 11.0$ years old; mean $\mathrm{HbA1c}$ level, $7.8 \pm 1.3 \%$; mean BMI, $26.6 \pm 4.1$; mean disease duration, $11.7 \pm 10.7$ years.

In all cases, liraglutide therapy was initiated prior to elective surgical procedures, which included orthopedic operations (40 cases); diagnostic or therapeutic cardiac catheterization for ischemic heart disease (12 cases); ophthalmological operations, including procedures for cataracts (13 cases); prostatectomy or prostate needle biopsy for prostate cancer ( 2 cases); mastectomy for breast cancer ( 1 case); unilateral nephrectomy for renal cancer (1 case); total hysterectomy for uterine cancer ( 1 case); total bladder extirpation for cancer (1 case); plastic surgery (1 case); and angioplasty for a hemodialysis shunt (1 case).
In the cases in which hyperglycemia occurred during the perioperative period, regular insulin was added at a dose that was appropriate to the patient's blood glucose level before each meal (Figure 1).

One patient with osteoarthritis of the knee was administered Liraglutide therapy but their operation was subsequently cancelled as they lost weight and their pain disappeared.

\section{Medication administered before the liraglutide therapy}

Regarding the medications administered before the liraglutide therapy, 14 patients did not receive any other medications before the liraglutide therapy, 43 patients were given oral medication, 27 patients were treated with sulfonylurea and. In the latter patients, the mean dose of glimepiride was $1.3 \pm 1.1 \mathrm{mg}$ per day. In addition, 16 patients were administered insulin (mean dose of insulin glargine: $10.6 \pm 5.0$ units per day).

\section{Glycemic control}

Compared with the values recorded on admission, the use of liraglutide improved the patients' preoperative blood glucose levels (Table 2). Furthermore, their postoperative blood glucose level sneaked at $250 \mathrm{mg} / \mathrm{dl}$.

The patients' mean glucose levels on the day on which liraglutide therapy was first administered, the day before the operation, the day of the operation, and the day after the operation were $198.0 \pm$ 47.5 (not including the patients that underwent ophthalmological operations), $144.2 \pm 26.5$ (not including the patients that underwent ophthalmological operations), $126.0 \pm 27.2$ (not including the patients that underwent cardiac catheterization for ischemic heart disease) ( $\mathrm{p}<0.01$ ), and $164.4 \pm 40.0$ (excluding the patients that underwent ophthalmological operations $)(p<0.01) \mathrm{mg} / \mathrm{dl}$, respectively. 
Citation: Katagiri N, Kigawa I, Yahara Y, Ueda Y, Kaneko S, et al. (2016) Liraglutide is a Perioperative Therapeutic Option for Patients with Type 2 Diabetes that Undergo Elective Surgery. Int J Diabetes Clin Diagn 3: 117. doi: http://dx.doi.org/10.15344/2394-1499/2016/117

Page 3 of 5

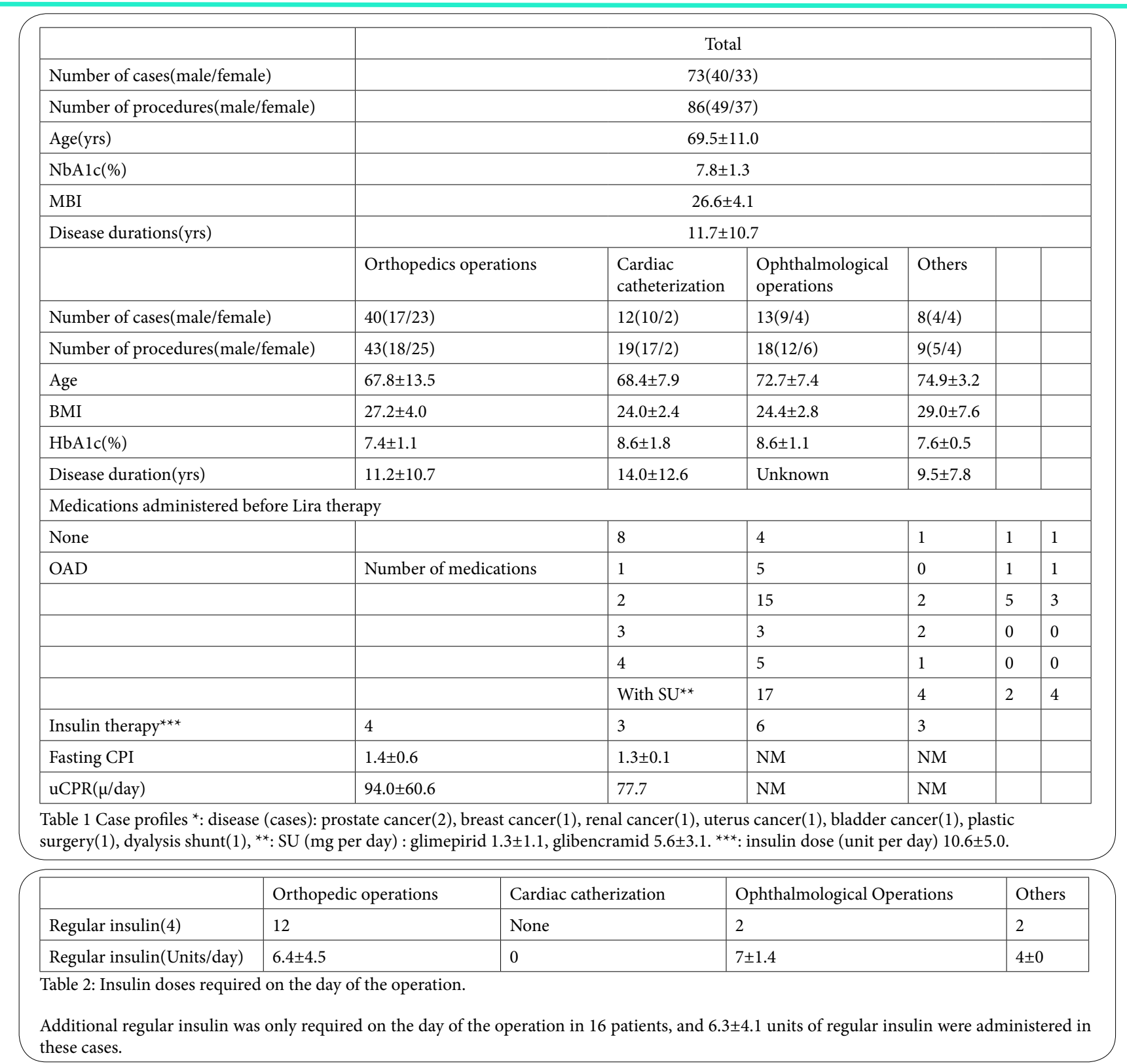

\section{Insulin doses prescribed during the perioperative period}

Additional regular insulin was only required on the day of the operation in 16 patients, and $6.3 \pm 4.1$ units of regular insulin were administered in these cases (Fiogure 2).

\section{Body weight and BMI}

The body weights of most of the patients decreased after the preoperative liraglrutide therapy until the day before the operation (mean: $-3.1 \pm 2.0 \mathrm{~kg}$ ), and the mean BMI change of the patients that received preoperative liraglutide therapy (except one orthopedic patient) was $-1.2 \pm 1.3$ (Figure 5).

The body weight loss induced by liraglutide therapy facilitated efficient rehabilitation.

\section{Hypoglycemic episodes and adverse events}

No adverse incidents such as hypoglycemic episodes, delayed wound healing, or other complications were observed in any of the cases.

\section{Others}

On the day of the operation, the patients' mean systolic blood pressure was $123.7 \pm 12.8 \mathrm{mmHg}$. The patients' HbA1c levels remained within the normal range $(6.8 \pm 0.8 \%)$ at 6 postoperative months.

\section{Discussion}

Insulin is the gold standard treatment for hyperglycemia in surgical patients. However, in the perioperative period, counterregulatory hormones, such as catecholamines, glucagon, cortisol, and growth hormones, are released as a response to stress, which causes insulin resistance and hepatic glucose production and results in hyperglycemia [6]. In addition, insulin therapy requires frequent glucose measurements and dose adjustment by well-trained medical providers, especially during the perioperative period, when patients' caloric intakes vary. The present results suggest that liraglutide can be 
Citation: Katagiri N, Kigawa I, Yahara Y, Ueda Y, Kaneko S, et al. (2016) Liraglutide is a Perioperative Therapeutic Option for Patients with Type 2 Diabetes that Undergo Elective Surgery. Int J Diabetes Clin Diagn 3: 117. doi: http://dx.doi.org/10.15344/2394-1499/2016/117

Page 4 of 5

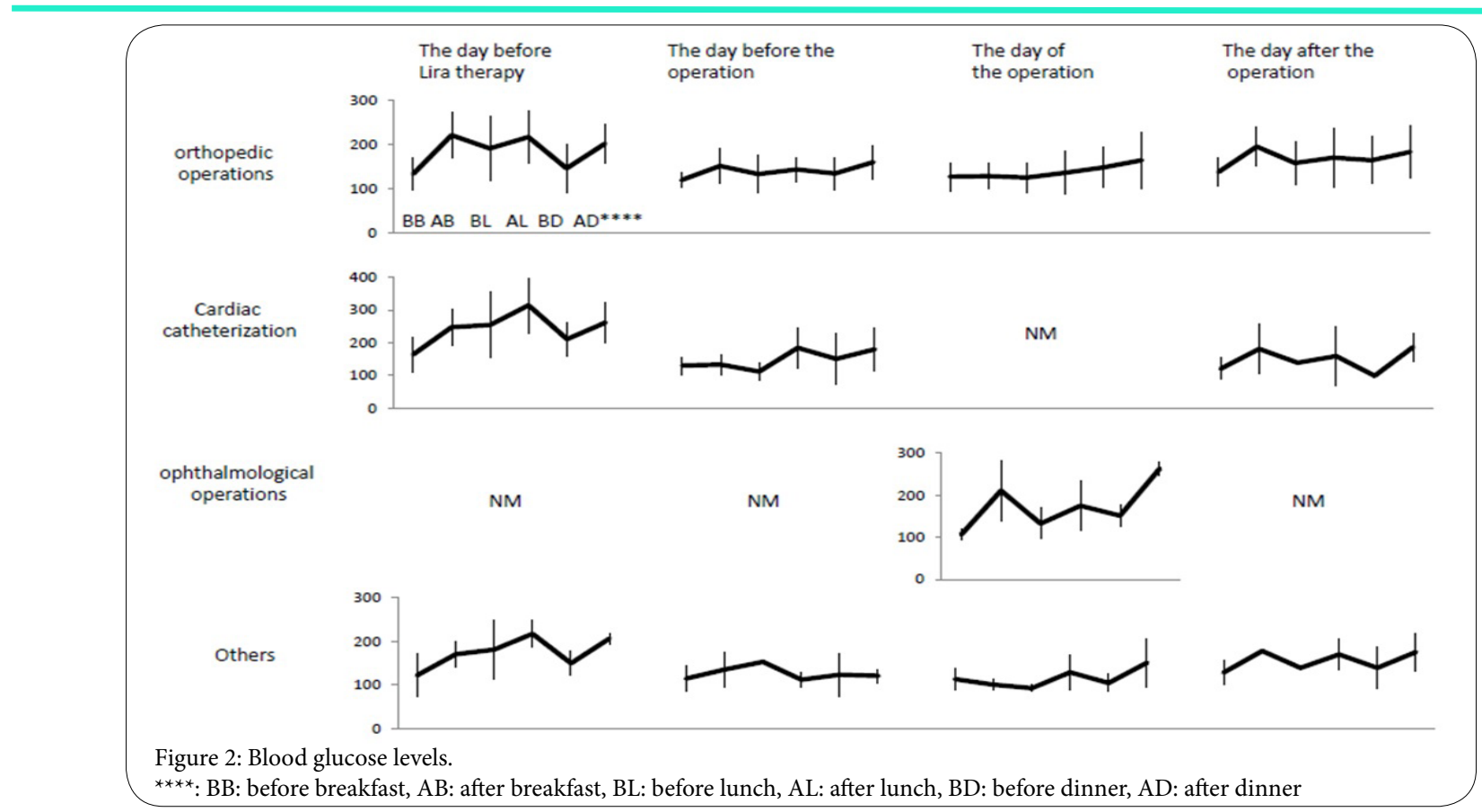

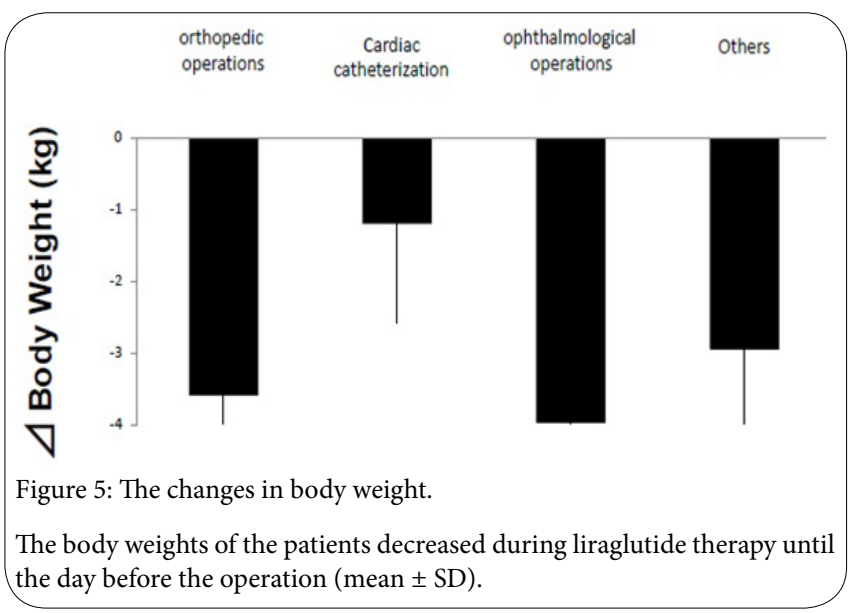

perioperatively administered to selected stable diabetic patients who are expected to consume meals at regular intervals without dose adjustment instead of insulin.

Hyperglucagonemia, which stimulates hepatic glucose production and causes severe insulin resistance in peripheral tissues [7], sometimes occurs in the postoperative period. Since liraglutide contributes to inhibiting glucagon secretion in the liver and improves hyperglycemia, we consider that it might be useful for reducing hyperglucagonemia in the postoperative period.

There are two further reasons why liraglutide, a GLP-1 agonist, is recommended as a therapeutic option for achieving glycemic control in elective perioperative patients. The first is that liraglutide is associated with a minimal risk of hypoglycemia [8]. Hypoglycemia is a serious complication of insulin therapy that is associated with a minimal risk of hypoglycemia [8]. Hypoglycemia is a serious complication of insulin therapy that is associated with many adverse cardiovascular events and has a negative impact on the mortality rate of hospitalized patients. In contrast, it has been reported that liraglutide has some beneficial effects on the cardiovascular system. Not only does it carry a low risk of hypoglycemia, but it also improves endothelial function, myocyte viability, and coronary artery vasodilation, etc. Considering these effects, liraglutide is useful especially in patients who are at high risk of ischemic heart disease $[9,10]$.

The second point is that liraglutide inhibits weight increases $[11,12]$. In the perioperative period, diabetic patients, especially those with limited exercise ability, e.g., patients undergoing orthopedic operations, tend to gain weight, and their glycemic control frequently deteriorates. As liraglutides lows gastric emptying, which attenuates postprandial glycemic variations, it also decreases appetite [13].The avoidance of weight gain before surgery leads to the maintenance of good glycemic control, even in the postoperative period. In addition, weight reductions would be expected to directly improve orthopedic conditions.

Clinicians should be aware of the following points: 1) As liraglutide is only available for patients whose $\beta$-cells secrete sufficient amounts of insulin, patients who are already dependent on insulin therapy or who have diabetic ketoacidosis or pancreatitis should be excluded from such treatment. 2) Moreover, as liraglutide decreases gastrointestinal motility, the use of liraglutide in patients that are scheduled to undergo gastrointestinal surgery should be avoided. 3) In cases in which postoperative hyperglycemia occurs, which is considered to be induced by overactive stress hormones, additional regular insulin should be considered to prevent postoperative complications, such as ketoacidosis, infection, and delayed wound healing occurring.

The limitations of the present study include the fact that it was conducted in a retrospective non-randomized manner, and so our findings need to be validated in a prospective, placebo-controlled study.

\section{Conclusion}

Liraglutide represents an effective therapeutic option for safely achieving good glycemic control in perioperative patients with T2DM, especially those who have a limited exercise ability who are at risk of hypoglycemia. 
Citation: Katagiri N, Kigawa I, Yahara Y, Ueda Y, Kaneko S, et al. (2016) Liraglutide is a Perioperative Therapeutic Option for Patients with Type 2 Diabetes that Undergo Elective Surgery. Int J Diabetes Clin Diagn 3: 117. doi: http://dx.doi.org/10.15344/2394-1499/2016/117

\section{References}

1. NICE-SUGAR Study Investigators, Finfer S, Chittock DR, Su SY, Blair D et al. (2009) Intensive versus conventional glucose control in critically ill patients. N Engl J Med 360: 1283-1297.

2. Bailey CJ, Tahrani AA, Barnett AH (2016) Future glucose-lowering drugs for type 2 diabetes. Lancet Diabetes Endocrinol 4: 350-359.

3. Schwartz S, DeFronzo RA (2013) Is incretin-based therapy ready for the care of hospitalized patients with type 2 diabetes?: The time has come for GLP-1 receptor agonists! Diabetes Care 36: 2107-2111.

4. Umpierrez GE, Korytkowski M (2013) Is Incretin-Based Therapy Ready for the Care of Hospitalized Patients With Type2 Diabetes? Insulin therapy has proven itself and is considered the mainstay of treatment. Diabetes Care 36: 2112-2117

5. Butler PC, Elashoff M, Elashoff R, Gale EA (2013) A critical analysis of the clinical use of incretin-based therapies: Are the GLP-1 therapies safe? Diabetes Care 36: 2118-2125

6. van Wezel HB, Zuurbier CJ, de Jonge E, van Dam EW, van Dijk J, et al. (2006) Differential effects of a perioperative hyperinsulinemic normoglycemic clamp on the neurohumoral stress response during coronary artery surgery. J Clin Endocrinol Metab 91: 4144-4153.

7. Christensen M, Bagger JI, Vilsbøll T, Knop FK (2011) The alpha-cell as target for type 2 diabetes therapy. Rev Diabet Stud 8: 369-381.

8. Seufert J, Gallwitz B (2014) The extra-pancreatic effects of GLP-1 receptor agonists: a focus on the cardiovascular, gastrointestinal and central nervous systems. Diabetes Obes Metab 16: 673-688.

9. McCormick LM, Heck PM, Ring LS, Kydd AC, Clarke SJ, et al. (2015) Glucagon-like peptide-1 protects against ischemic left ventricular dysfunction during hyperglycemia in patients with coronary artery disease and type 2 diabetes mellitus. Cardiovasc Diabetol 14: 102-114.

10. Mima A (2016) Incretin-Based Therapy for Prevention of Diabetic Vascular Complications. J Diabetes Res 2016: 1379274.

11. de Wit HM, Vervoort GM, Jansen HJ, de Galan BE, Tack CJ (2016) Durable efficacy of liraglutide in patients with type 2 diabetes and pronounced insulin-associated weight gain: 52-week results from the Effect of Liraglutide on insulin-associated wEight GAiN in patients with Type 2 diabetes' (ELEGANT) randomized controlled trial. J Intern Med 279: 283-292.

12. Perna S, Guido D, Bologna C, Solerte SB, Guerriero F, et al. (2016) Liraglutide and obesity in elderly: efficacy in fat loss and safety in order to prevent sarcopenia. A perspective case series study. Aging Clin Exp Res 26749118.

13. Meier JJ, Rosenstock J, Hincelin-Méry A, Roy-Duval C, Delfolie A, et al. (2015) Contrasting effects of lixisenatide and liraglutide on postprandial glycemic control, gastric emptying, and safety parameters in patients with type 2 diabetes on optimized insulin glargine with or without metformin: A randomized, open-label trial. Diabetes Care 38:1 263-1273. 